\title{
Global Asymptotic Stability in a Class of Reaction-Diffusion Equations with Time Delay
}

\author{
Yueding Yuan ${ }^{1,2,3}$ and Zhiming Guo ${ }^{1,3}$ \\ ${ }^{1}$ School of Mathematics and Information Sciences, Guangzhou University, Guangzhou 510006, China \\ ${ }^{2}$ School of Mathematics and Computer Sciences, Yichun University, Yichun 336000, China \\ ${ }^{3}$ Key Laboratory of Mathematics and Interdisciplinary Science of Guangdong Higher Education Institutes, \\ Guangzhou University, Guangzhou 510006, China
}

Correspondence should be addressed to Zhiming Guo; gzm100@sina.com

Received 19 February 2014; Accepted 8 April 2014; Published 29 April 2014

Academic Editor: Chuangxia Huang

Copyright (C) 2014 Y. Yuan and Z. Guo. This is an open access article distributed under the Creative Commons Attribution License, which permits unrestricted use, distribution, and reproduction in any medium, provided the original work is properly cited.

We study a very general class of delayed reaction-diffusion equations in which the reaction term can be nonmonotone and spatially nonlocal. By using a fluctuation method, combined with the careful analysis of the corresponding characteristic equations, we obtain some sufficient conditions for the global asymptotic stability of the trivial solution and the positive steady state to the equations subject to the Neumann boundary condition.

\section{Introduction}

There has been a growing interest in the dynamic behavior of spatial nonlocal and time-delayed population systems since the 1970s [1]. When the death function of such a system is linear many researchers used the theory of monotone semiflows, the comparison arguments, and the fluctuation method to study spreading speeds, traveling waves, and the global stability (see, e.g., [2-10]). However, researches on these problems become relatively rare for nonmonotone delayed reaction-diffusion systems in which the death function is nonlinear (see [11, 12]). The reason lies in the fact that it is difficult to establish an appropriate expression for solutions to study the solution semiflow under this case.

In this paper, we will investigate the global asymptotic stability of the positive steady state for the following timedelayed reaction-diffusion equation:

$$
\begin{aligned}
\frac{\partial w(t, x)}{\partial t}= & d \Delta w(t, x)-f(w(t, x)) \\
& +\int_{\Omega} k(\alpha, x, y) b(w(t-\tau, y)) d y,
\end{aligned}
$$

$$
\begin{gathered}
\frac{\partial w(t, x)}{\partial \mathbf{n}}=0, \quad t>0, x \in \partial \Omega, \\
w(t, x)=\phi(t, x) \geq 0, \quad t \in[-\tau, 0], x \in \Omega,
\end{gathered}
$$

where $d>0, \alpha \geq 0, \tau \geq 0, \Delta$ denotes the Laplacian operator on $\mathbb{R}^{m}, \Omega$ is a bounded and open domain of $\mathbb{R}^{m}$ with a smooth boundary $\partial \Omega, \partial / \partial \mathbf{n}$ is the differentiation in the direction of the outward normal $\mathbf{n}$ to $\partial \Omega$, and the kernel function $k(\alpha, x, y)$ is given by

$$
k(\alpha, x, y)= \begin{cases}\sum_{n=1}^{+\infty} e^{-\lambda_{n} \alpha} \varphi_{n}(x) \varphi_{n}(y), & \text { if } \alpha>0, \\ \delta(x-y), & \text { if } \alpha=0 .\end{cases}
$$

Here, $0=\lambda_{1}<\lambda_{2} \leq \cdots \leq \lambda_{n} \leq \cdots$ with $\lim _{n \rightarrow \infty} \lambda_{n}=+\infty$ is the eigenvalue of the linear operator $-\Delta$ subject to the homogeneous Neumann boundary condition on $\partial \Omega, \varphi_{n}$ is the eigenvector corresponding to $\lambda_{n},\left\{\varphi_{n}\right\}_{n=1}^{+\infty}$ is a complete orthonormal system in the space $L^{2}(\bar{\Omega}), \varphi_{1}(x)>0$ for all $x \in$ $\Omega$, and $\delta(x)$ is the Dirac function on $\mathbb{R}^{m}[10,13]$. Throughout 
this paper, we assume that the functions $f$ and $b$ satisfy the following.

(A1) $b: \mathbb{R}^{+} \rightarrow \mathbb{R}^{+}$is Lipschitz continuous with $b(0)=0$ and $b^{\prime}(0)>0$, and $b(w) \leq b^{\prime}(0) w$ for all $w \geq 0$.

(A2) $f(w)=w g(w)$ for all $w \geq 0$, where $g: \mathbb{R}^{+} \rightarrow \mathbb{R}^{+}$ is Lipschitz continuous with $g^{\prime}(0) \geq 0, g(w)>0$, and $g^{\prime}(w) \geq 0$ for all $w>0$.

(A3) There exists a positive number $M$ such that, for all $w>$ $M, \bar{b}(w)<f(w)$, where $\bar{b}(w)=\max _{u \in[0, w]} b(u)$.

In the monotone case, where the function $b(w)$ increases with $w>0, \mathrm{Xu}$ and Zhao [12] studied the global dynamics of (1) and obtained some results on the uniqueness and global attractivity of a positive steady state by using the theory of monotone dynamical systems. In the case of $f(w)=$ $\mu w$, Zhao [10] proved the global attractivity of the positive constant equilibrium for (1) by using a fluctuation method of Thieme and Zhao [14], where $\mu$ is a positive constant. In the case where $f(w)=\mu w$ and $\alpha=0$, Yi and Zou [7] proved the global attractivity of the unique positive constant equilibrium for (1) by combining a dynamical systems argument and some subtle inequalities. In the case where $\Omega=[0, L]$ and $f(w)=\mu w$, (1) reduces to the equation derived in [3], where the numerical solutions are considered. A global convergence theorem was obtained in [11] for a special case of (1).

The aim of this paper is to establish some criteria to guarantee the global asymptotic stability of the trivial solution and the positive steady state for (1) by using a fluctuation method, combined with the careful analysis of the corresponding characteristic equations. The interesting thing is that main results obtained in this paper extend the related existing results.

The rest of this paper is organized as follows. We will present some preliminary results in Section 2. Our main results are presented and proved in Sections 3 and 4, where we obtain sufficient conditions to ensure the global asymptotic stability of the trivial solution and the positive steady state for (1) in a nonmonotone case. In Section 5, we provide four examples to illustrate the applicability of the main results.

\section{Preliminaries}

Firstly, we show that the kernel $k(\alpha, x, y)$ in (2) enjoys the following properties.

Lemma 1. For $\alpha>0$, one has

(i) $\left.(\partial / \partial \mathbf{n}) k(\alpha, x, y)\right|_{x \in \partial \Omega}=\left.(\partial / \partial \mathbf{n}) k(\alpha, x, y)\right|_{y \in \partial \Omega}=0$,

(ii) $0<k(\alpha, x, y) \leq C^{*}$, for all $x, y \in \Omega$, where $C^{*}$ is a positive constant depending only on $m$ and $\Omega$,

(iii) $\left|\varphi_{n}(x)\right| \leq \sqrt{C^{*}} \exp \left[(1 / 2) \lambda_{n} \alpha\right]$, for all $x \in \Omega, n=$ $1,2, \ldots$,

(iv) $\int_{\bar{\Omega}} k(\alpha, x, y) d y=1$, for all $x \in \Omega$.

Proof. The verification of (i) is straightforward and is thus omitted. Part (ii) follows from [15, Lemma 3.2.1 and Theorem 4.4.6] since $k(\alpha, x, y)$ is a heat kernel of the heat equation
$(\Delta-(\partial / \partial \alpha)) u(x, \alpha)=0$. Part (iii) follows from $e^{-\lambda_{n} \alpha}\left(\varphi_{n}(x)\right)^{2} \leq k(\alpha, x, x) \leq C^{*}$, for all $x \in \Omega, n=1,2, \ldots$. And part (iv) follows from $\lambda_{1}=0, \varphi_{1}(x) \equiv \sqrt{1 / \operatorname{mes}(\Omega)}$, and $\int_{\bar{\Omega}} \varphi_{n}(y) d y=0$ for all $n=2,3, \ldots$, where $\operatorname{mes}(\Omega)$ is the measure of $\Omega$. The proof is completed.

Let $\mathbb{X}=C(\bar{\Omega}, \mathbb{R})$ and $\mathbb{X}^{+}=\{\phi \in \mathbb{X} \mid \phi(x) \geq 0, \forall x \in \bar{\Omega}\}$. Then $\left(\mathbb{X}, \mathbb{X}^{+}\right)$is a strongly ordered Banach space. It is well known that the differential operator $A=d \Delta$ generates a $C^{0}$-semigroup $T(t)$ on $\mathbb{X}$. Moreover, the standard parabolic maximum principle (see, e.g., [16, Corollary 7.2.3]) implies that the semigroup $T(t): \mathbb{X} \rightarrow \mathbb{X}$ is strongly positive in the sense that $T(t)\left(\mathbb{X}^{+} \backslash\{0\}\right) \subset \operatorname{Int}\left(\mathbb{X}^{+}\right), \forall t>0$.

Let $\mathbb{Y}=C([-\tau, 0], \mathbb{X})$ and $\mathbb{Y}^{+}=C\left([-\tau, 0], \mathbb{X}^{+}\right)$. For the sake of convenience, we will identify an element $\phi \in \mathbb{Y}$ as a function from $[-\tau, 0] \times \bar{\Omega}$ to $\mathbb{R}$ defined by $\phi(s, x)=\phi(s)(x)$, and for each $s \in[-\tau, 0]$, we regard $f(\phi(s))$ as a function on $\bar{\Omega}$ defined by $f(\phi(s))=f(\phi(s, \cdot))$. For any function $w(\cdot)$ : $[-\tau, \sigma) \rightarrow \mathbb{X}$, where $\sigma>0$, we define $w_{t} \in \mathbb{Y}, t \in[0, \sigma)$ by $w_{t}(s)=w(t+s), \forall s \in[-\tau, 0]$. Define $F: \mathbb{Y}^{+} \rightarrow \mathbb{X}$ by

$$
\begin{array}{r}
F(\phi)(x)=-f(\phi(0, x))+\int_{\bar{\Omega}} k(\alpha, x, y) b(\phi(-\tau, y)) d y \\
\forall x \in \bar{\Omega}, \phi \in \mathbb{Y}^{+} .
\end{array}
$$

Then we can rewrite (1) as an abstract functional equation:

$$
\begin{gathered}
\frac{d w(t)}{d t}=A w(t)+F\left(w_{t}\right), \quad t \geq 0 \\
w_{0}=\phi \in \mathbb{Y}^{+} .
\end{gathered}
$$

Therefore, we can write (4) as an integral equation:

$$
\begin{gathered}
w(t)=T(t) \phi(0)+\int_{0}^{t} T(t-s) F\left(w_{s}\right) d s, \quad t \geq 0, \\
w_{0}=\phi \in \mathbb{Y}^{+}
\end{gathered}
$$

whose solutions are called mild solutions for (1).

Since $T(t): \mathbb{X} \rightarrow \mathbb{X}$ is strongly positive, we have

$$
\lim _{h \rightarrow 0^{+}} \operatorname{dist}\left(\phi(0)+h F(\phi), \mathbb{X}^{+}\right)=0, \quad \forall \phi \in \mathbb{Y}^{+}
$$

By [17, Proposition 3 and Remark 2.4] (or [18, Corollary 8.1.3]), for each $\phi \in \mathbb{Y}^{+}$, (1) has a unique noncontinuable mild solution $w(t, \phi)$ with $w_{0}=\phi$, and $w(t, \phi) \in \mathbb{X}^{+}$for all $t \in\left(0, \sigma_{\phi}\right)$. Moreover, $w(t, \phi)$ is a classical solution of (1) for $t>\tau$ (see [18, Corollary 2.2.5]).

By the same arguments as in the proof of [12, Theorems 2.1 and 3.1], we have the following two lemmas.

Lemma 2. Let $(A 1)-(A 3)$ hold. Then, for each $\phi \in \mathbb{Y}^{+}$, a unique solution $w(t, \phi)$ of (1) globally exists on $[-\tau, \infty)$, $\limsup _{t \rightarrow \infty} w(t, x, \phi) \leq M$ uniformly for $x \in \bar{\Omega}$, and the solution semiflow $\Phi(t)=w_{t}(\cdot): \mathbb{Y}^{+} \rightarrow \mathbb{Y}^{+}, t \geq 0$, admits a connected global attractor. 
Lemma 3. Let (A1)-(A3) hold, and let $w(t, x, \phi)$ be the solution of (1) with $\phi \in \mathbb{Y}^{+}$. Then the following two statements are valid.

(i) If $b^{\prime}(0)<g(0)$, then for any $\phi \in \mathbb{Y}^{+}$, we have $\limsup _{t \rightarrow \infty} w(t, x, \phi)=0$ uniformly for $x \in \bar{\Omega}$.

(ii) If $b^{\prime}(0)>g(0)$, then (1) admits at least one spatially homogeneous steady state $w^{*} \in(0, M]$, and there exists $\eta>0$ such that for any $\phi \in \mathbb{Y}^{+}$with $\phi(0, \cdot) \neq \equiv$ we have $\liminf _{t \rightarrow \infty} w(t, x, \phi) \geq \eta$ uniformly for $x \in \bar{\Omega}$.

Note that in case (ii) above, the function $S(w)=b(w)-$ $f(w)$ satisfies $S(0)=0, S^{\prime}(0)>0$, and $S(M) \leq 0$. Therefore, there exists at least one positive number $w^{*} \in(0, M]$ such that $S\left(w^{*}\right)=0$, and hence, $w^{*}$ is a spatially homogeneous steady state of (1).

\section{Global Attractivity}

In this section, we establish the global attractivity of the positive and spatially homogeneous steady state $w^{*}$ for (1) by the fluctuation method used in [10, Theorem 3.1].

Motivated by [10, Section 3], we assume further that the functions $f(w)$ and $b(w)$ satisfy the following.

(A4) $b^{\prime}(0)>g(0),(b(w) / f(w))$ is strictly decreasing for $w \in(0, M]$, and $f(w)$ and $b(w)$ have the property (P) that, for any $u, v \in(0, M]$ satisfying $u \leq w^{*} \leq v$, $f(u) \geq b(v)$, and $f(v) \leq b(u)$, we have $u=v$.

Note that if $b(w)$ is nondecreasing for $w \in[0, M]$, then $f(w)$ and $b(w)$ have the property $(P)$. Indeed, for any $0<u \leq$ $w^{*} \leq v \leq M$ with $f(u) \geq b(v)$ and $f(v) \leq b(u)$, we have

$$
f\left(w^{*}\right) \leq f(v) \leq b(u) \leq b\left(w^{*}\right) \leq b(v) \leq f(u) \leq f\left(w^{*}\right),
$$

which implies that $u=v=w^{*}$. Combining this observation and [10, Lemma 3.1] with $\mu w$ replaced by $f(w)$, where $\mu>0$, we then have the following result.

Lemma 4. Either of the following two conditions is sufficient for the property $(P)$ in condition (A4) to hold.

(P1) $b(w)$ is nondecreasing for $w \in[0, M]$.

(P2) $f(w) b(w)$ is strictly increasing for $w \in(0, M]$.

Now we are in a position to prove our main result in this section.

Theorem 5. Assume that (A1)-(A4) hold, and let $w(t, x, \phi)$ be the solution of (1) with $\phi \in \mathbb{Y}^{+}$. Then for any $\phi \in \mathbb{Y}^{+}$with $\phi(0, \cdot) \neq \equiv$, we have $\lim _{t \rightarrow \infty} w(t, x, \phi)=w^{*}$ uniformly for $x \in \bar{\Omega}$.

In order to prove Theorem 5, we will need the following lemma.
Lemma 6. Assume that (A1)-(A3) hold, and let $w(t, x) \equiv$ $w(t, x, \phi)$ be the solution of (1) with $\phi \in \mathbb{Y}^{+}$. Then $w(t, x)$ satisfies

$$
\begin{aligned}
& w(t, x) \\
& =e^{-\gamma t} \int_{\Omega} k(d t, x, y) \phi(0, y) d y \\
& +\int_{0}^{t} e^{-\gamma s} \int_{\Omega} k(d s, x, y) \\
& \times[\gamma w(t-s, y)-f(w(t-s, y)) \\
& \left.+\int_{\Omega} k(\alpha, y, z) b(w(t-s-\tau, z)) d z\right] d y d s,
\end{aligned}
$$

where $\gamma=\max _{w \in[0, M]} f^{\prime}(w)$ and the kernel function $k$ is given in (2).

Proof. Let

$$
\begin{aligned}
H(t, x) \equiv & \gamma w(t, x)-f(w(t, x)) \\
& +\int_{\Omega} k(\alpha, x, y) b(w(t-\tau, y)) d y .
\end{aligned}
$$

Since $\mathbb{X} \subset L^{2}(\bar{\Omega})$, for each $t \geq 0$, there exist real numbers $a_{n}(t)$ and $b_{n}(t), n=1,2, \ldots$, such that

$$
\begin{aligned}
& w(t, x)=\sum_{n=1}^{+\infty} a_{n}(t) \varphi_{n}(x), \\
& H(t, x)=\sum_{n=1}^{+\infty} b_{n}(t) \varphi_{n}(x) .
\end{aligned}
$$

Therefore, by (10), (11), and (1), we have

$$
\begin{gathered}
a_{n}(0)=\int_{\Omega} \phi(0, y) \varphi_{n}(y) d y, \\
b_{n}(s)=\int_{\Omega} H(s, y) \varphi_{n}(y) d y, \\
\frac{d a_{n}(t)}{d t}=-\left(d \lambda_{n}+\gamma\right) a_{n}(t)+b_{n}(t), \quad n=1,2, \ldots
\end{gathered}
$$

By using the variation of constants method, we obtain

$$
\begin{array}{r}
a_{n}(t)=\left[a_{n}(0)+\int_{0}^{t} e^{\left(d \lambda_{n}+\gamma\right) s} b_{n}(s) d s\right] e^{-\left(d \lambda_{n}+\gamma\right) t}, \\
n=1,2, \ldots
\end{array}
$$


Thus, by (10), (12), and (14), we further get

$$
\begin{aligned}
w & (t, x) \\
= & \sum_{n=1}^{+\infty}\left[a_{n}(0)+\int_{0}^{t} e^{\left(d \lambda_{n}+\gamma\right) s} b_{n}(s) d s\right] e^{-\left(d \lambda_{n}+\gamma\right) t} \varphi_{n}(x) \\
= & e^{-\gamma t} \sum_{n=1}^{+\infty} \int_{\Omega} \phi(0, y) e^{-d \lambda_{n} t} \varphi_{n}(x) \varphi_{n}(y) d y \\
& +\int_{0}^{t} e^{-\gamma(t-s)} \sum_{n=1}^{+\infty} \int_{\Omega} e^{-d \lambda_{n}(t-s)} H(s, y) \varphi_{n}(x) \varphi_{n}(y) d y d s \\
= & e^{-\gamma t} \int_{\Omega} \phi(0, y) k(d t, x, y) d y \\
& +\int_{0}^{t} e^{-\gamma(t-s)} \int_{\Omega} H(s, y) k(d(t-s), x, y) d y d s \\
= & e^{-\gamma t} \int_{\Omega}^{t} \phi(0, y) k(d t, x, y) d y \\
& +\int_{0}^{t} e^{-\gamma s} \int_{\Omega} H(t-s, y) k(d s, x, y) d y d s .
\end{aligned}
$$

Therefore, (8) follows immediately from (9) and (15). The proof is completed.

Proof of Theorem 5. For any given $\phi \in \mathbb{Y}^{+}$with $\phi(0, \cdot) \not \equiv 0$, let $\omega(\phi)$ be the omega limit set of the positive orbit through $\phi$ for the solution semiflow $\Phi(t)$. By Lemma 1, we get $\omega(\phi) \subset$ $\mathbb{A} \subseteq \mathbb{Y}_{[0, M]}$, where $\mathbb{A}$ is the global attractor of the solution semiflow $\Phi(t)$ and

$$
\mathbb{Y}_{[0, M]} \equiv\{\phi \in \mathbb{Y} \mid 0 \leq \phi(\theta, x) \leq M, \forall(\theta, x) \in[-\tau, 0] \times \bar{\Omega}\} .
$$

Note that $\mathbb{A}$ is a maximal compact invariant set of the solution semiflow $\Phi(t)$. Thus, it is sufficient to prove the global attractivity of $w^{*}$ for all $\phi \in \mathbb{Y}_{[0, M]}$ with $\phi(0, \cdot) \not \equiv 0$.

Let $\phi \in \mathbb{Y}_{[0, M]}$ be given such that $\phi(0, \cdot) \not \equiv 0$. Then it follows from Lemma 6 that

$$
\begin{aligned}
& w(t, x) \\
& =e^{-\gamma t} \int_{\Omega} k(d t, x, y) \phi(0, y) d y \\
& +\int_{0}^{t} e^{-\gamma s} \int_{\Omega} k(d s, x, y) \\
& \times[\gamma w(t-s, y)-f(w(t-s, y)) \\
& \left.\quad+\int_{\Omega} k(\alpha, y, z) b(w(t-s-\tau, z)) d z\right] d y d s,
\end{aligned}
$$

where $w(t, x) \equiv w(t, x, \phi)$ is the solution of (1) starting from the initial function $\phi$. Following [19], we define a function $h$ : $[0, M] \times[0, M] \rightarrow \mathbb{R}$ by

$$
h(u, v)= \begin{cases}\min \{b(w) \mid u \leq w \leq v\}, & \text { if } u \leq v, \\ \max \{b(w) \mid v \leq w \leq u\}, & \text { if } v \leq u .\end{cases}
$$

Then $h(u, v)$ is nondecreasing in $u \in[0, M]$ and nonincreasing in $v \in[0, M]$. Moreover, $b(w)=h(w, w), \forall w \in[0, M]$, and $h(u, v)$ is continuous in $(u, v) \in[0, M] \times[0, M]$ (see $[20$, Section 2]). Therefore, by (17), we have

$$
\begin{aligned}
& w(t, x) \\
& =e^{-\gamma t} \int_{\Omega} k(d t, x, y) \phi(0, y) d y \\
& \quad+\int_{0}^{t} e^{-\gamma s} \int_{\Omega} k(d s, x, y) \\
& \quad \times[\gamma w(t-s, y)-f(w(t-s, y)) \\
& \quad+\int_{\Omega} k(\alpha, y, z) \\
& \quad \times h(w(t-s-\tau, z), w(t-s-\tau, z)) d z] d y d s .
\end{aligned}
$$

Let

$$
\begin{array}{r}
w^{\infty}(x) \equiv \limsup _{t \rightarrow \infty} w(t, x), \quad w_{\infty}(x) \equiv \liminf _{t \rightarrow \infty} w(t, x), \\
\forall x \in \bar{\Omega} .
\end{array}
$$

Then Lemmas 2 and 3 imply that

$$
M \geq w^{\infty}(x) \geq w_{\infty}(x) \geq \eta>0, \quad \forall x \in \bar{\Omega} .
$$

On the other hand, note that $\gamma=\max _{w \in[0, M]} f^{\prime}(w)$. Therefore, the function $\gamma w-f(w)$ is nondecreasing in $w \in[0, M]$. Thus, by Fatou's lemma and (19), we further get

$$
\begin{aligned}
& w^{\infty}(x) \\
& \leq \int_{0}^{\infty} e^{-\gamma s} \int_{\Omega} k(d s, x, y) \\
& \times\left[\gamma w^{\infty}(y)-f\left(w^{\infty}(y)\right)\right. \\
& \left.+\int_{\Omega} k(\alpha, y, z) h\left(w^{\infty}(z), w_{\infty}(z)\right) d z\right] d y d s .
\end{aligned}
$$

Let

$$
w^{\infty} \equiv \sup _{x \in \bar{\Omega}} w^{\infty}(x), \quad w_{\infty} \equiv \inf _{x \in \bar{\Omega}} w_{\infty}(x)
$$

Then

$$
M \geq w^{\infty} \geq w_{\infty} \geq \eta>0 .
$$


Moreover, it follows from Lemma 1 that

$$
\begin{array}{r}
\int_{\Omega} k(d s, x, y) d y=1, \quad \int_{\Omega} k(\alpha, x, y) d y=1, \\
\forall s \geq 0, \quad x \in \bar{\Omega} .
\end{array}
$$

Therefore, by (22), we have

$$
\begin{aligned}
w^{\infty} & \leq\left[\gamma w^{\infty}-f\left(w^{\infty}\right)+h\left(w^{\infty}, w_{\infty}\right)\right] \int_{0}^{\infty} e^{-\gamma s} d s \\
& =\frac{1}{\gamma}\left[\gamma w^{\infty}-f\left(w^{\infty}\right)+h\left(w^{\infty}, w_{\infty}\right)\right] .
\end{aligned}
$$

Thus,

$$
f\left(w^{\infty}\right) \leq h\left(w^{\infty}, w_{\infty}\right) .
$$

Similarly, we have

$$
f\left(w_{\infty}\right) \geq h\left(w_{\infty}, w^{\infty}\right) .
$$

By (18), we may find $u, v \in\left[w_{\infty}, w^{\infty}\right] \subset(0, M]$ such that

$$
h\left(w^{\infty}, w_{\infty}\right)=b(u), \quad h\left(w_{\infty}, w^{\infty}\right)=b(v) .
$$

It then follows from (27) and (28) that

$$
b(u) \geq f\left(w^{\infty}\right) \geq f(u), \quad b(v) \leq f\left(w_{\infty}\right) \leq f(v),
$$

and hence,

$$
\frac{b(v)}{f(v)} \leq 1=\frac{b\left(w^{*}\right)}{f\left(w^{*}\right)} \leq \frac{b(u)}{f(u)} .
$$

This, together with the strict monotonicity of $b(w) / f(w)$ for $w \in(0, M]$, implies that $u \leq w^{*} \leq v$. Moreover, by (27) and (28), we also have

$$
b(u) \geq f\left(w^{\infty}\right) \geq f(v), \quad b(v) \leq f\left(w_{\infty}\right) \leq f(u) .
$$

Therefore, the property $(\mathrm{P})$ implies that

$$
u=v=w^{*} \text {. }
$$

Thus, by (30), we obtain

$$
w^{\infty}=w_{\infty}=w^{*} .
$$

Since

$$
w^{\infty} \geq w^{\infty}(x) \geq w^{\infty}(x) \geq w^{\infty}, \quad x \in \bar{\Omega},
$$

we further get

$$
w^{\infty}(x)=w_{\infty}(x)=w^{*}, \quad x \in \bar{\Omega} .
$$

This implies that

$$
\lim _{t \rightarrow \infty} w(t, x)=w^{*}, \quad x \in \bar{\Omega} .
$$

It remains to prove that $\lim _{t \rightarrow \infty} w(t, x)=w^{*}$ uniformly for $x \in \bar{\Omega}$. For any $\psi \in \omega(\phi)$, there exists a sequence $t_{n} \rightarrow \infty$ such that $\Phi\left(t_{n}\right) \phi \rightarrow \psi$ in $\mathbb{Y}$ as $n \rightarrow \infty$. Therefore, we have

$$
\lim _{n \rightarrow \infty} w\left(t_{n}+\theta, x, \phi\right)=\psi(\theta, x)
$$

uniformly for $(\theta, x) \in[-\tau, 0] \times \bar{\Omega}$. By (37), we further get

$$
\psi(\theta, x)=w^{*}, \quad \forall(\theta, x) \in[-\tau, 0] \times \bar{\Omega} .
$$

Thus, we obtain $\omega(\phi)=\left\{w^{*}\right\}$, which implies that $w(t, \cdot, \phi)$ converges to $w^{*}$ in $\mathbb{X}$ as $t \rightarrow \infty$. The proof is completed.

\section{Global Asymptotic Stability}

In this section, we establish the global asymptotic stability of the trivial solution and the positive and spatially homogeneous steady state $w^{*}$ for (1) by the careful analysis of the corresponding characteristic equations. To this end, we first give the following formal definitions of stability (see, e.g., [18, Remark 2.1.3]).

Definition 7. Let $w=\widehat{w}$ be a steady state of the abstract equation (4). It is called stable if for any $\varepsilon>0$ there exists $\delta>0$ such that the solution $w(t, \phi)$ of $(4)$ with $\|\phi-\widehat{w}\|_{\mathbb{Y}}<$ $\delta$ satisfies $\|w(t, \phi)-\widehat{w}\|_{\mathbb{X}}<\varepsilon$, for all $t \geq 0$. It is called unstable if it is not stable. It is asymptotically stable if it is stable and there exists $\delta_{0}>0$ such that the solution $w(t, \phi)$ of (4) with $\|\phi-\widehat{w}\|_{\mathbb{Y}}<\delta_{0}$ satisfies $\lim _{t \rightarrow+\infty}\|w(t, \phi)-\widehat{w}\|_{\mathbb{X}}=$ 0 . It is globally asymptotically stable if it is stable and any solution $w(t, \phi)$ of (4) with arbitrary $\phi \in \mathbb{Y}$ satisfies $\lim _{t \rightarrow+\infty}\|w(t, \phi)-\widehat{w}\|_{\mathbb{X}}=0$.

Let $\widehat{w}$ be a spatially homogeneous steady state for (1) (e.g., the trivial solution and $w^{*}$ ). Define $G: \mathbb{Y}^{+} \rightarrow \mathbb{X}$ by

$$
\begin{aligned}
& G(\phi)(x)=-f^{\prime}(\widehat{w}) \phi(0, x) \\
&+b^{\prime}(\widehat{w}) \int_{\bar{\Omega}} k(\alpha, x, y) \phi(-\tau, y) d y, \\
& \forall x \in \bar{\Omega}, \quad \phi \in \mathbb{Y}^{+},
\end{aligned}
$$

where $f^{\prime}(\widehat{w})=\left.(d f(w) / d w)\right|_{w=\widehat{w}}$ and $b^{\prime}(\widehat{w})=(d b(w) /$ $d w)\left.\right|_{w=\widehat{w}}$. Note that $k(\alpha, x, y)$ is given in (2). Then we can write the linearized equation of (1) at $w=\widehat{w}$ as the following abstract functional equation

$$
\begin{gathered}
\frac{d w(t)}{d t}=A w(t)+G\left(w_{t}\right), \quad t \geq 0, \\
w_{0}=\phi \in \mathbb{Y}^{+},
\end{gathered}
$$

where $A$ can be referred to Section 2 .

For each complex number $\lambda$ we define the $\mathbb{X}$-valued linear operator $\Theta(\lambda)$ by

$$
\Theta(\lambda) u=A u-\lambda u+G\left(e^{\lambda} u\right), \quad u \in \operatorname{Dom}(A),
$$

where $e^{\lambda \cdot} u \in \mathbb{Y}$ is defined by (note that we use $\mathbb{Y}$ to denote its complexification here)

$$
\left(e^{\lambda \cdot} u\right)(\theta)=e^{\lambda \theta} u, \quad \theta \in[-\tau, 0]
$$

We will call $\lambda$ a characteristic value of (41) if there exists $u \in$ $\operatorname{Dom}(A) \backslash\{0\}$ solving the characteristic equation $\Theta(\lambda) u=0$ (see, e.g., [18]). Since $\operatorname{Dom}(A) \subset \mathbb{X} \subset L^{2}(\bar{\Omega})$, for any $u \in$ $\operatorname{Dom}(A) \backslash\{0\}$, there exist complex numbers $a_{n}, n=1,2, \ldots$, such that

$$
u(x)=\sum_{n=1}^{+\infty} a_{n} \varphi_{n}(x)
$$


Therefore, by (2), (42), and (44), we have

$$
\begin{aligned}
\Theta & (\lambda) u(x) \\
= & d \Delta u(x)-\lambda u(x)-f^{\prime}(\widehat{w}) u(x) \\
& +b^{\prime}(\widehat{w}) \int_{\bar{\Omega}} e^{-\tau \lambda} u(y) k(\alpha, x, y) d y \\
= & \sum_{n=1}^{+\infty} a_{n}\left[-d \lambda_{n}-\lambda-f^{\prime}(\widehat{w})+b^{\prime}(\widehat{w}) e^{-\tau \lambda} e^{-\lambda_{n} \alpha}\right] \varphi_{n}(x) .
\end{aligned}
$$

Thus, the characteristic value $\lambda$ of (41) satisfies at least one of the following equations:

$$
\lambda=-d \lambda_{n}-f^{\prime}(\widehat{w})+b^{\prime}(\widehat{w}) e^{-\lambda_{n} \alpha} e^{-\tau \lambda}, \quad n=1,2, \ldots
$$

Lemma 8. Assume that (A1)-(A3) hold, and let $\beta$ be the smallest real number such that if $\lambda$ is a characteristic value of (41), then $\operatorname{Re} \lambda \leq \beta$. One has the following:

(i) if $b^{\prime}(\widehat{w})>f^{\prime}(\widehat{w})$, then $\beta>0$,

(ii) if $-f^{\prime}(\widehat{w}) \leq b^{\prime}(\widehat{w})<f^{\prime}(\widehat{w})$, then $\beta<0$,

(iii) if $b^{\prime}(\widehat{w})=f^{\prime}(\widehat{w})$, then $\beta=0$.

Proof. (i) If $b^{\prime}(\widehat{w})>f^{\prime}(\widehat{w})$, then, by (46) and [21, Proposition 4.6], there exists at least one characteristic value $\lambda$ of (41) such that $\operatorname{Re} \lambda>0$. Therefore, $\beta>0$.

(ii) If $-f^{\prime}(\widehat{w}) \leq b^{\prime}(\widehat{w})<f^{\prime}(\widehat{w})$, then since $0=\lambda_{1}<\lambda_{2} \leq$ $\cdots \leq \lambda_{n} \leq \cdots$, we have

$$
\begin{aligned}
-\left[d \lambda_{n}+f^{\prime}(\widehat{w})\right] e^{\lambda_{n} \alpha} & \leq b^{\prime}(\widehat{w}) \\
& <\left[d \lambda_{n}+f^{\prime}(\widehat{w})\right] e^{\lambda_{n} \alpha}, \quad n=1,2, \ldots
\end{aligned}
$$

Therefore, by (46) and [21, Proposition 4.6], all the characteristic values of (41) have negative real parts. Thus, it follows from [18, Theorem 3.1.10] that $\beta<0$.

(iii) If $b^{\prime}(\widehat{w})=f^{\prime}(\widehat{w})$, then $\lambda=0$ is a characteristic value of (41). Therefore, $\beta \geq 0$. If $\beta>0$, then there exists at least one characteristic value of (41) $\lambda^{(0)}$ and a positive number $n$ such that $\operatorname{Re} \lambda^{(0)}>0$ and

$$
\lambda^{(0)}=-d \lambda_{n}-f^{\prime}(\widehat{w})+b^{\prime}(\widehat{w}) e^{-\lambda_{n} \alpha} e^{-\tau \lambda^{(0)}} .
$$

Let $\lambda^{(0)}=x^{(0)}+i y^{(0)}$, where $x^{(0)}$ and $y^{(0)}$ both are real numbers. Then $x^{(0)}>0$. By (48), we have

$$
x^{(0)}=-d \lambda_{n}-f^{\prime}(\widehat{w})+f^{\prime}(\widehat{w}) e^{-\lambda_{n} \alpha} e^{-\tau x^{(0)}} \cos \left(\tau y^{(0)}\right),
$$

and hence, $\cos \left(\tau y^{(0)}\right)>0$. This implies that

$$
\begin{aligned}
x^{(0)} & \leq-f^{\prime}(\widehat{w})+f^{\prime}(\widehat{w}) e^{-\tau x^{(0)}} \cos \left(\tau y^{(0)}\right) \\
& =f^{\prime}(\widehat{w})\left[e^{-\tau x^{(0)}} \cos \left(\tau y^{(0)}\right)-1\right] .
\end{aligned}
$$

But, since $x^{(0)}>0$, we have $e^{-\tau x^{(0)}} \cos \left(\tau y^{(0)}\right)<1$. Therefore,

$$
x^{(0)} \leq f^{\prime}(\widehat{w})\left[e^{-\tau x^{(0)}} \cos \left(\tau y^{(0)}\right)-1\right]<0
$$

contradicting $x^{(0)}>0$. This contradiction proves $\beta=0$. The proof is completed.

Now we are ready to summarize our main results on the global stability. By Definition 7, Lemmas 3 and 8, Theorem 5, [18, Corollary 3.1.11], and the principle of linearized stability (see, e.g., [21]), we obtain the following.

Theorem 9. Assume that (A1)-(A3) hold. Then the following two statements are valid.

(i) If $b^{\prime}(0)<g(0)$, then the zero solution of (1) is globally asymptotically stable in $\mathbb{Y}^{+}$.

(ii) If $b^{\prime}(0)>g(0)$, then the zero solution of (1) is unstable, and (1) admits at least one spatially homogeneous steady state $w^{*} \in(0, M]$.

Theorem 10. Assume that (A1)-(A3) hold, and $b^{\prime}(0)>g(0)$. Then the following two statements for the positive and spatially homogeneous steady state $w^{*}$ of (1) are valid.

(i) If $b^{\prime}\left(w^{*}\right)>f^{\prime}\left(w^{*}\right)$, then $w^{*}$ is unstable.

(ii) If $-f^{\prime}\left(w^{*}\right) \leq b^{\prime}\left(w^{*}\right)<f^{\prime}\left(w^{*}\right)$ and (A4) hold, then $w^{*}$ is globally asymptotically stable in $\mathbb{Y}^{+} \backslash\{0\}$.

\section{Examples}

In this section, we present four examples to illustrate the feasibility of our main results.

Example 1. Consider the equation resulting from letting $f(w)=\mu w$ in (1); that is,

$$
\begin{aligned}
& \frac{\partial w(t, x)}{\partial t}=d \Delta w(t, x)-\mu w(t, x) \\
& \quad+\int_{\Omega} k(\alpha, x, y) b(w(t-\tau, y)) d y, \\
& \frac{\partial w(t, x)}{\partial \mathbf{n}}=0, \quad t>0, x \in \partial \Omega, \\
& w(t, x)=\phi(t, x) \geq 0, \quad t \in[-\tau, 0], x \in \Omega,
\end{aligned}
$$

where $\mu$ is a positive constant.

In this case, we now formulate the following assumptions to replace $(\mathrm{A} 2)-(\mathrm{A} 4)$ :

$\left(\mathrm{A} 2^{\prime}\right)$ There exists a positive number $M$ such that, for all $w>$ $M, \bar{b}(w)<\mu w$, where $\bar{b}(w)=\max _{u \in[0, w]} b(u)$.

$\left(\mathrm{A} 3^{\prime}\right) b^{\prime}(0)>\mu,(b(w) / w)$ is strictly decreasing for $w \epsilon$ $(0, M]$, and $b(w)$ has the property $\left(P^{\prime}\right)$ that, for any $u, v \in(0, M]$ satisfying $u \leq w^{*} \leq v, \mu u \geq b(v)$, and $\mu v \leq b(u)$, we have $u=v$. 
By applying Theorems 9 and 10, we then obtain the following results for (52).

Theorem 11. Assume that (A1) and $\left(A 2^{\prime}\right)$ hold. Then the following two statements are valid.

(i) If $b^{\prime}(0)<\mu$, then the zero solution of (52) is globally asymptotically stable in $\mathbb{Y}^{+}$.

(ii) If $b^{\prime}(0)>\mu$, then the zero solution of (52) is unstable, and (52) admits at least one spatially homogeneous steady state $w^{*} \in(0, M]$.

Theorem 12. Assume that (A1) and $\left(A 2^{\prime}\right)$ hold, and $b^{\prime}(0)>\mu$. Then the following two statements for the positive and spatially homogeneous steady state $w^{*}$ of (52) are valid.

(i) If $b^{\prime}\left(w^{*}\right)>\mu$, then $w^{*}$ is unstable.

(ii) If $-\mu \leq b^{\prime}\left(w^{*}\right)<\mu$ and $\left(A 3^{\prime}\right)$ hold, then $w^{*}$ is globally asymptotically stable in $\mathbb{Y}^{+} \backslash\{0\}$.

Remark 13. It is easy to see that (52) is discussed in [10] and some partial results of Theorems 11 and 12 have been obtained [10].

Example 2. Consider the following Nicholson's blowfly equation resulting from letting $f(w)=\mu w$ and $b(w)=p w e^{-q w}$ in (1):

$$
\begin{aligned}
& \frac{\partial w(t, x)}{\partial t}= d \Delta w(t, x)-\mu w(t, x) \\
&+\int_{\Omega} p w(t-\tau, y) e^{-q w(t-\tau, y)} k(\alpha, x, y) d y, \\
& \frac{\partial w(t, x)}{\partial \mathbf{n}}=0, \quad t>0, x \in \partial \Omega, \\
& w(t, x)=\phi(t, x) \geq 0, \quad t \in[-\tau, 0], x \in \Omega,
\end{aligned}
$$

where $p$ and $q$ are two positive constants.

By the same arguments as in [10, Section 4], together with Theorems 11 and 12, we have the following results for (53).

Theorem 14. (i) If $p<\mu$, then the zero solution of (53) is globally asymptotically stable in $\mathbb{Y}^{+}$.

(ii) If $p>\mu$, then the zero solution of (53) is unstable, and (53) admits the unique positive constant equilibrium $w^{*}=$ $(\ln (p / \mu)) / q$.

Theorem 15. If $\mu<p \leq e^{2} \mu$, the unique positive constant equilibrium $w^{*}=(\ln (p / \mu)) / q$ of $(53)$ is globally asymptotically stable in $\mathbb{Y}^{+} \backslash\{0\}$.

Remark 16. It is easy to see that the equation in [7] is a special case of $\alpha=0$ of (53). Hence all main results of [7] are special cases of our Theorems 14 and 15.
Example 3. Consider the following Mackey-Glass equation resulting from letting $f(w)=\mu w^{l+1}$ and $b(w)=p w /\left(q+w^{l}\right)$ in (1):

$$
\begin{aligned}
& \frac{\partial w(t, x)}{\partial t}=d \Delta w(t, x)-\mu w^{l+1}(t, x) \\
& \quad+\int_{\Omega} \frac{p w(t-\tau, y)}{q+w^{l}(t-\tau, y)} k(\alpha, x, y) d y, \\
& \frac{\partial w(t, x)}{\partial \mathbf{n}}=0, \quad t>0, x \in \partial \Omega, \\
& w(t, x)=\phi(t, x) \geq 0, \quad t \in[-\tau, 0], x \in \Omega,
\end{aligned}
$$

where $l$ is a positive constant.

By the same arguments as in [10, Section 4], together with Theorems 9 and 10, we have the following results for (54).

Theorem 17. The zero solution of (54) is always unstable, and (54) must admit the unique positive constant equilibrium $w^{*}=$ $\left(Z_{0}\right)^{1 / l}$, where

$$
Z_{0}=\frac{1}{2}\left(-q+\sqrt{q^{2}+\frac{4 p}{\mu}}\right)
$$

Theorem 18. If $\left(\left(p q+p(1-l) Z_{0}\right) /\left(q+Z_{0}\right)^{2}\right)>\mu(1+l) Z_{0}$, the unique positive constant equilibrium $w^{*}=\left(Z_{0}\right)^{1 / l}$ is unstable, and if $-\mu(1+l) Z_{0} \leq\left(\left(p q+p(1-l) Z_{0}\right) /\left(q+Z_{0}\right)^{2}\right)<\mu(1+l) Z_{0}$ it is globally asymptotically stable in $\mathbb{Y}^{+} \backslash\{0\}$.

Example 4. Consider the equation resulting from letting $f(w)=\mu w^{2}$ and $b(w)=p w(1-(w / r))$ in (1); that is,

$$
\begin{aligned}
& \frac{\partial w(t, x)}{\partial t} \\
& =d \Delta w(t, x)-\mu w^{2}(t, x) \\
& +\int_{\Omega} p w(t-\tau, y)\left(1-\frac{w(t-\tau, y)}{r}\right) k(\alpha, x, y) d y, \\
& \quad \frac{\partial w(t, x)}{\partial \mathbf{n}}=0, \quad t>0, x \in \partial \Omega, \\
& w(t, x)=\phi(t, x) \geq 0, \quad t \in[-\tau, 0], x \in \Omega,
\end{aligned}
$$

where $0<r \leq+\infty$.

Clearly, $w^{*}=\left(p /\left(\mu+p r^{-1}\right)\right), \max _{w>0} b(w)=b(r / 2)$, and (A1)-(A3) hold, where $r^{-1}=0$ if $r=+\infty$. Moreover, $0<$ $w^{*} \leq r / 2$ if $\mu \geq p r^{-1}$. Therefore, (A4) is satisfied if $\mu \geq p r^{-1}$. Thus, Theorems 9 and 10 imply the following results.

Theorem 19. The zero solution of (56) is always unstable, and (56) must admit the unique positive constant equilibrium $w^{*}=$ $\left(p /\left(\mu+p r^{-1}\right)\right)$. 
Theorem 20. If $\mu \geq p r^{-1}$, then the unique positive constant equilibrium $w^{*}=\left(p /\left(\mu+p r^{-1}\right)\right)$ is globally asymptotically stable in $\mathbb{Y}^{+} \backslash\{0\}$.

Remark 21. It is easy to see that the equation in [11] is a special case of $r=+\infty$ of (56) and hence some partial results of Theorems 19 and 20 have been obtained [11].

\section{Conflict of Interests}

The authors declare that there is no conflict of interests regarding the publication of this paper.

\section{Acknowledgments}

This work was partially supported by National Natural Science Foundation of China, Research Fund for the Doctoral Program of Higher Education of China (no. 20124410110001), and Program for Changjiang Scholars and Innovative Research Team in University (IRT1226).

\section{References}

[1] S. A. Gourley and J. Wu, "Delayed non-local diffusive systems in biological invasion and disease spread," in Nonlinear Dynamics and Evolution Equations, H. Brunner, X.-Q. Zhao, and X. Zou, Eds., vol. 48 of Fields Institute Communications, pp. 137-200, American Mathematical Society, Providence, RI, USA, 2006.

[2] C. Huang, Z. Yang, T. Yi, and X. Zou, "On the basins of attraction for a class of delay differential equations with nonmonotone bistable nonlinearities," Journal of Differential Equations, vol. 256, no. 7, pp. 2101-2114, 2014.

[3] D. Liang, J. W.-H. So, F. Zhang, and X. Zou, "Population dynamic models with nonlocal delay on bounded domains and their numerical computations," Differential Equations and Dynamical Systems, vol. 11, no. 1-2, pp. 117-139, 2003.

[4] J. W.-H. So, J. Wu, and Y. Yang, "Numerical steady state and Hopf bifurcation analysis on the diffusive Nicholson's blowflies equation," Applied Mathematics and Computation, vol. 111, no. 1, pp. 33-51, 2000.

[5] J. W.-H. So, J. Wu, and X. Zou, "A reaction-diffusion model for a single species with age structure. I. Travelling wavefronts on unbounded domains," The Royal Society of London Proceedings A: Mathematical, Physical and Engineering Sciences, vol. 457, no. 2012, pp. 1841-1853, 2001.

[6] H. R. Thieme and X.-Q. Zhao, "Asymptotic speeds of spread and traveling waves for integral equations and delayed reactiondiffusion models," Journal of Differential Equations, vol. 195, no. 2, pp. 430-470, 2003.

[7] T. Yi and X. Zou, "Global attractivity of the diffusive Nicholson blowflies equation with Neumann boundary condition: a nonmonotone case," Journal of Differential Equations, vol. 245, no. 11, pp. 3376-3388, 2008.

[8] T. Yi and X. Zou, "Global dynamics of a delay differential equation with spatial non-locality in an unbounded domain," Journal of Differential Equations, vol. 251, no. 9, pp. 2598-2611, 2011.

[9] T. Yi and X. Zou, "On Dirichlet problem for a class of delayed reaction-diffusion equations with spatial non-locality," Journal of Dynamics and Differential Equations, vol. 25, no. 4, pp. 959979, 2013.
[10] X.-Q. Zhao, "Global attractivity in a class of nonmonotone reaction-diffusion equations with time delay," Canadian Applied Mathematics Quarterly, vol. 17, no. 1, pp. 271-281, 2009.

[11] S. A. Gourley and Y. Kuang, "Wavefronts and global stability in a time-delayed population model with stage structure," The Royal Society of London Proceedings A: Mathematical, Physical and Engineering Sciences, vol. 459, no. 2034, pp. 1563-1579, 2003.

[12] D. Xu and X.-Q. Zhao, "A nonlocal reaction-diffusion population model with stage structure," Canadian Applied Mathematics Quarterly, vol. 11, no. 3, pp. 303-319, 2003.

[13] L. C. Evans, Partial Differential Equations, vol. 19 of Graduate Studies in Mathematics, American Mathematical Society, Providence, RI, USA, 1998.

[14] H. R. Thieme and X.-Q. Zhao, "A non-local delayed and diffusive predator-prey model," Nonlinear Analysis: Real World Applications, vol. 2, no. 2, pp. 145-160, 2001.

[15] S. T. Yau and R. Schoen, Lectures on Differential Geometry, Higher Education Press, Beijing, China, 1994.

[16] H. L. Smith, Monotone Dynamical Systems: An Introduction to the Theory of Competitive and Cooperative Systems, vol. 41 of Mathematical Surveys and Monographs, American Mathematical Society, Providence, RI, USA, 1995.

[17] R. H. Martin, Jr. and H. L. Smith, "Abstract functionaldifferential equations and reaction-diffusion systems," Transactions of the American Mathematical Society, vol. 321, no. 1, pp. $1-44,1990$.

[18] J. Wu, Theory and Applications of Partial Functional-Differential Equations, vol. 119 of Applied Mathematical Sciences, Springer, New York, NY, USA, 1996.

[19] H. R. Thieme, "Density-dependent regulation of spatially distributed populations and their asymptotic speed of spread," Journal of Mathematical Biology, vol. 8, no. 2, pp. 173-187, 1979.

[20] H. R. Thieme, "On a class of Hammerstein integral equations," Manuscripta Mathematica, vol. 29, no. 1, pp. 49-84, 1979.

[21] H. Smith, An Introduction to Delay Differential Equations with Applications to the Life Sciences, vol. 57 of Texts in Applied Mathematics, Springer, New York, NY, USA, 2011. 


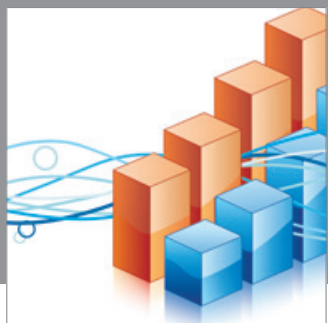

Advances in

Operations Research

mansans

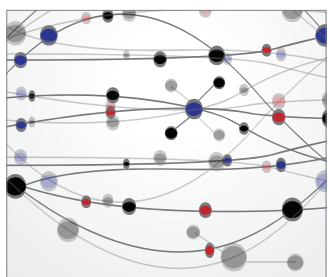

The Scientific World Journal
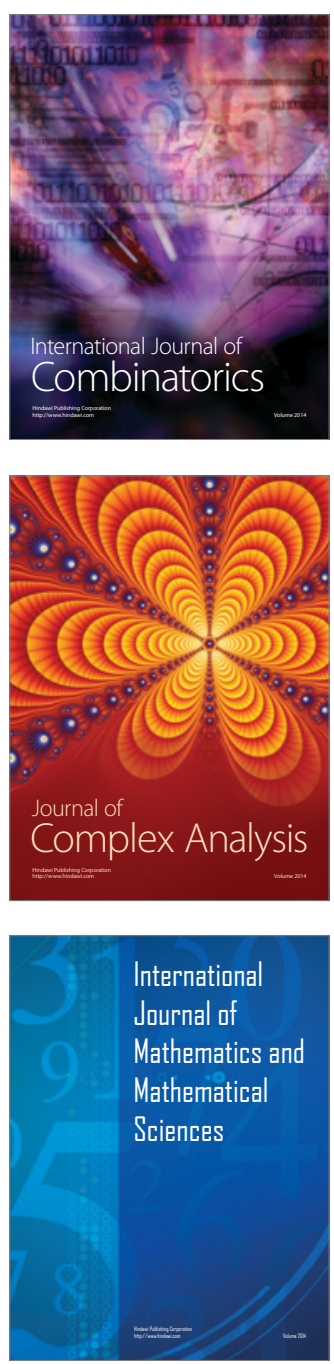
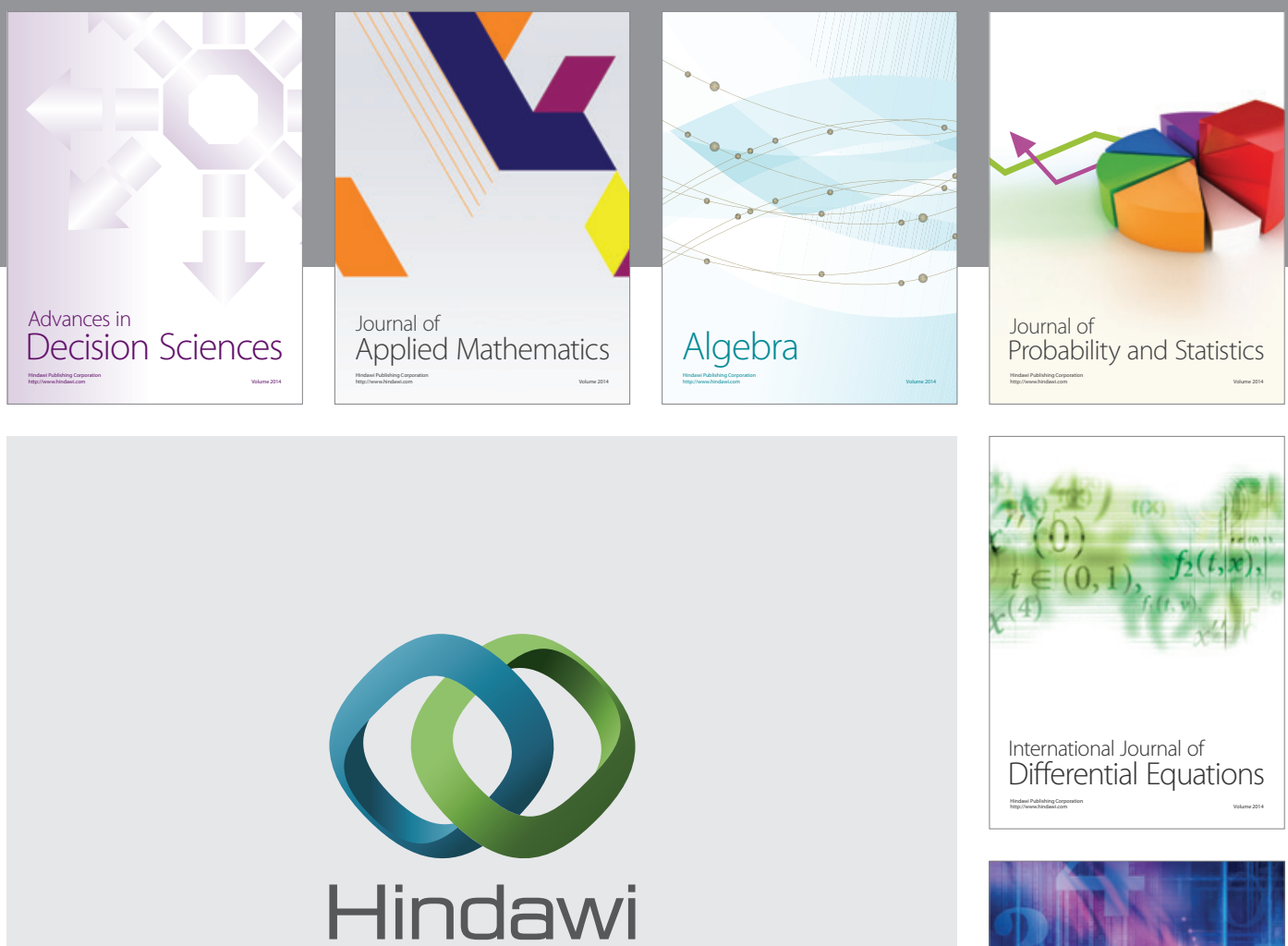

Submit your manuscripts at http://www.hindawi.com
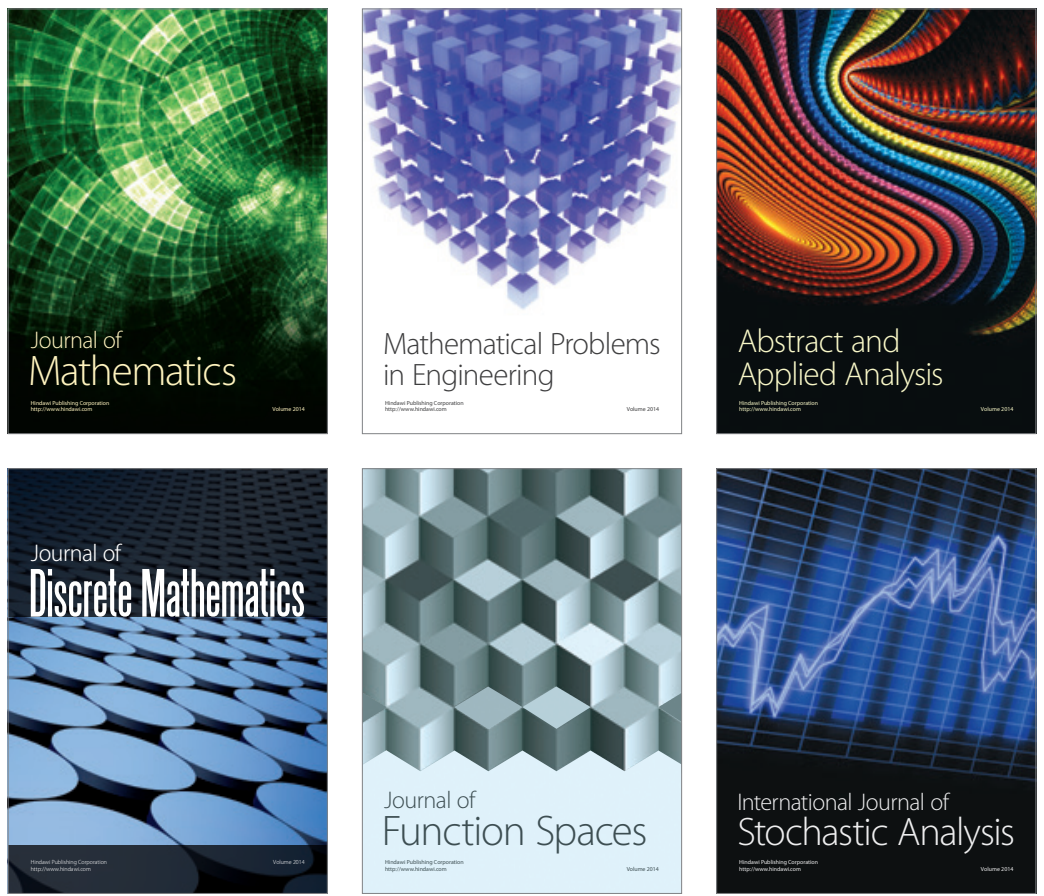

Journal of

Function Spaces

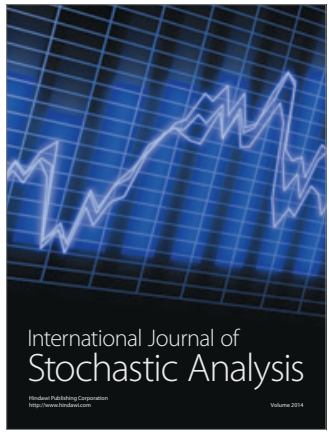

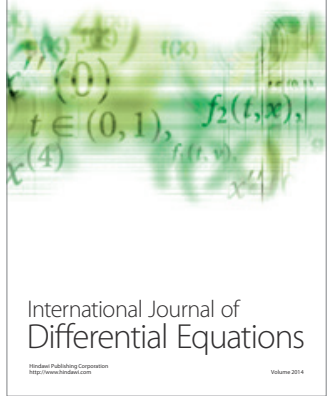
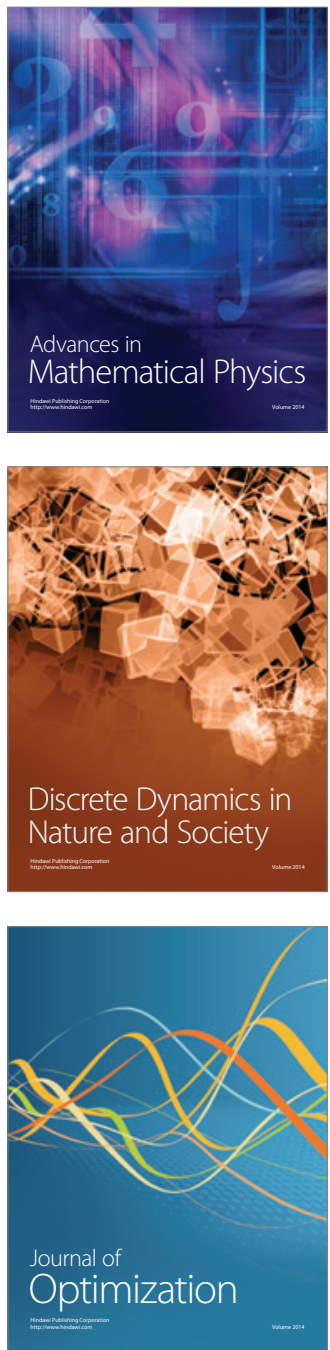